\title{
Harms and benefits: Collecting ethnicity data in a clinical context
}

\author{
Colleen Varcoe*, Annette J. Browne, Sabrina Wong, Victoria L. Smye \\ School of Nursing, University of British Columbia, 2211 Wesbrook Mall, Vancouver, BC, Canada V6T $2 B 5$
}

\section{A R T I C L E I N F O}

\section{Article history:}

Available online 13 March 2009

\section{Keywords:}

Canada

Race/ethnicity

Data collection

Ethics

Inequity

Health disparities

Discrimination

\begin{abstract}
A B S T R A C T
Although ethnicity data are collected in most countries at the population level, it has become more common to collect such data in healthcare settings, partially in response to growing health and social inequities worldwide. However, the implications of doing so have not been studied. This two-year study was designed to critically examine the implications of collecting ethnicity data in healthcare settings. Using a critical ethnographic approach, we interviewed 104 patients, community and healthcare leaders, and healthcare workers within diverse clinical contexts in a large city in Western Canada in 2006-2007. This paper presents an interpretive thematic analysis, using an ethical lens, of the harms and benefits associated with the process of data collection in a clinical context. While most leaders and healthcare workers and some patients envisioned potential benefits associated with having ethnicity data, these benefits were seen as largely contingent upon action being taken to ameliorate inequities. Overwhelmingly, however, leaders from ethno-cultural communities and patients of diverse identities anticipated potential harm arising both from having ethnicity data and the process of collection. The analysis illustrates that in today's sociopolitical context, collecting ethnicity data in clinical contexts may engender considerable harm, particularly for racialized, vulnerable patients. If ethnicity data are currently collected at the population level, evidence of benefit is required before proceeding to collect these data at the point of care.
\end{abstract}

(c) 2009 Elsevier Ltd. All rights reserved.

\section{Introduction}

Interest in collecting data regarding ethnicity has grown in recent years, partially in response to growing social and health inequities worldwide. In many countries, ethnicity data are routinely collected at the population level through self-report surveys such as the National Health Interview Survey in the United States (National Center for Health Statistics, 2008). In healthcare settings, collecting data on patients' ethnicity has become routine in places such as the United Kingdom, United States, and New Zealand. The main rationale is to enable identification of and provide a basis for rectifying inequities in order to achieve more equitable and culturally competent care (see for example, King et al., 2008; New Zealand Ministry of Health, 2004; Sheth et al., 1997; Smedley, Stith, \& Nelson, 2002). Ethnicity data are typically collected as part of administrative data, or at the point of care, that is, when people seek care at physicians' offices, hospitals or community health centers.

\footnotetext{
* Corresponding author. Tel.: +1 6048273121.

E-mail address: colleen.varcoe@nursing.ubc.ca (C. Varcoe).
}

Unlike other countries, in Canada, information on ethnicity is generally not collected in healthcare contexts (Rummens, 2003). Exceptions occur in certain agencies that seek information for their own records, particularly to identify First Nations people (one group of Aboriginal people in Canada) to whom specific policies may apply. No federal, provincial, or other governmental mandates require healthcare institutions or agencies to collect data on ethnicity. Therefore, most Canadian research on ethnicity and health uses large population-based surveys as sources of ethnicity data. However, the meaningfulness of Canadian ethnicity data has been much debated (Bourhis, 2003; Jedwab, 2003; Rummens, 2003) with an increasing proportion of the population identifying "Canadian" as their ethnicity on census data, reflecting the extent to which ethnicity and identity are fluid constructs shaped by individual and sociopolitical contexts (Thomas, 2005).

Statistics Canada (2006) acknowledges ethnicity as an ambiguous concept because it can encompass multiple different aspects such as "race," country of origin or ancestry, identity, language and religion, changing from context to context as a result of new immigration flows and the development of new identities. In healthcare contexts (as in other sectors of society), ethnicity is often conflated with "race", contributing to a narrow and erroneous categorical conceptualization of ethnicity. For example, in the USA, 
ethnicity is used interchangeably with race, contributing to the mistaken assumption that these are biological and/or genetic rather than social categories (Krieger, 2004). The notion that race is a valid, biological, meaningful, a priori category persists despite the decades of scientific evidence from population genetics, social epidemiology, anthropology and sociology that calls race into question as an essential and meaningful set of biological categories (Krieger, 1999a; United Nations, 1952). This is not to deny the significance of ethnicity or race as social categories, and the effects of racial discrimination or ethnic stereotyping on health or access to healthcare. Problematic, however, is the way ethnicity - as a multifaceted concept - has been constructed narrowly for the purposes of data collection and analysis in health research.

Despite these limitations of categorization, healthcare organizations in some parts of Canada are increasingly focused on collecting ethnicity data as a way of monitoring which groups are accessing services and potentially reducing access inequities and making services more culturally relevant. This was the case in one Western province, where a Health Authority (a regional body responsible for healthcare delivery) planned to collect ethnicity data at clinical points of contact. Prior to implementation, representatives of the Health Authority joined with university researchers to examine critically the implications of collecting ethnicity data in clinical contexts. As one aspect of our analysis, we used an ethical lens to consider the potential harms and benefits. The purpose of this paper is to report that analysis.

\section{Background to the study}

Over the past two decades researchers have made compelling calls to examine variables such as ethnicity, race, and socioeconomic status that underlie persistent inequities (see, for example, Bhopal, 2001; Krieger, 1999b; New Zealand Ministry of Health, 2004; Sheth et al., 1997; Smedley et al., 2002; Williams, 2002; Wu \& Schimmele, 2005). Within a research context, these and other authors argue that progress or setbacks in addressing racial and ethnic inequalities in health cannot be monitored without population-level racial and ethnic data (Krieger, 2000; Krieger et al., 2005). Although some take this position while cautioning that collecting race and ethnicity data is a "double edged sword" (Krieger, 2004), others argue for elimination of racial and ethnic categories in data collection, as classifying people by race and ethnicity tacitly reinforces racial and ethnic divisions in society (Bhopal, 1998; Kaplan \& Bennett, 2003).

Beyond the research context, there has been a trend toward collecting ethnicity data within healthcare agencies and institutions. For example, in the USA where ethnicity data have been collected for decades, the Institute of Medicine (IOM), Physicians for Human Rights, and The Commonwealth Fund support the collection of standardized data on race, ethnicity, and primary language of patients within healthcare organizations (HasnainWynia \& Baker, 2006; Hasnain-Wynia, Pierce, \& Pittman, 2004; King et al., 2008; Perot \& Youdelman, 2001; Physicians for Human Rights, 2003; Smedley et al., 2002). This has been suggested primarily a) to identify and mitigate existing health disparities by facilitating the provision of culturally and linguistically appropriate healthcare, and b) to promote quality healthcare for all population groups by contributing to better information databases (New Zealand Ministry of Health, 2004; Sheth et al., 1997; Smedley et al., 2002). According to the IOM, "a critical barrier to eliminating disparities and improving the quality of patient care is the frequent lack of even the most basic data on race, ethnicity or primary language of patients within healthcare organizations" (HasnainWynia et al., 2004, p. v). The assumption is that providing equitable, quality care requires race and ethnicity data, but to date research has not shown that knowledge about health inequities leads to better healthcare services at the individual level. Indeed in the UK, Aspinall (2000) found that little use was made of ethnicity data collected in healthcare settings. Aspinall and Anionwu (2002) reported that although the ethnic identities of patients were obtained for an annual total in England of over 11 million admissions, the information has only been used to produce indices of quality, not to improve the quality of care provided. The possible reasons for such failure include that the contribution of racism and causes of inequities is complex, encompassing structural inequities and institutional racism (Bhopal, 2007) and that additional resources required to address such inequities may not be prioritized in the context of global healthcare reforms that have emphasized cost cutting.

Given growing interest in ethnicity data in healthcare contexts and interest in collecting such data, the general public and patients have been surveyed regarding their attitudes toward ethnicity data collection. Quan, Wong, Johnson, and Ghali (2006) found that of 2799 respondents to a telephone survey randomly selected from those with listed phone numbers in a Western Canadian city, 84.8\% felt comfortable about recording their ethnicity in hospital charts. This finding did not vary by ethnicity, however, $73 \%$ of the sample identified as "white" and there was a $45 \%$ non-response rate. In the United Kingdom, Pringle and Rothera (1996) found that $72 \%$ of patients thought that general practitioners "definitely" or "possibly" should record ethnicity, but the response rate was 56\% and the ethnic identity of respondents was not published. In the United States, Baker et al. (2005) found that of 220 patients in a general internal medical setting about $80 \%$ somewhat or strongly agreed that healthcare providers should collect information on patients' race/ethnicity. However, $28 \%$ had significant discomfort reporting their own race/ethnicity, and 58\% (including three quarters of the African American participants) were somewhat or very concerned the information could be used to discriminate against patients. Although these surveys provide a glimpse into the complexities involved, to date, the possible harms and benefits of collecting ethnicity data at the point of care have received little attention and have not been examined in Canada.

Collecting ethnicity data in a clinical context, particularly in Canada where such data have not been collected previously, has different features than collecting data in the context of vital statistics data, a national survey or census, or in other populationbased research contexts. In healthcare contexts, ethnicity tends to be conceptualized very narrowly, and is often used as synonymous with "race" (Anderson \& Kirkham, 1998; Bhopal, 2001; Ford \& Kelly, 2005; Gerrish, 2000). This is problematic in terms of data collection, given the ambiguity of the concept. Further, research provides mounting evidence of ethnic and racial discrimination and structural racism in healthcare (e.g. Anderson \& Kirkham, 1998; Balsa, McGuire, \& Meredith, 2005; Bhopal, 2007; Bourassa, McKayMcNabb, \& Hampton, 2004; Henry \& Tator, 2006). Thus, the implications of collecting ethnicity data within such contexts are not known.

\section{Research methods}

In order to explore the implications of collecting ethnicity data in healthcare settings, we employed a critical ethnographic design and collected data in four modes in a large Western Canadian city: (a) in-depth interviews with 10 decision-makers and policy leaders affiliated with several health authorities and policy research units; (b) three focus groups of community leaders $(n=18)$ from a range of ethno-cultural groups who served on committees of the Health Authority to represent patients' perspectives concerning healthcare planning; (c) semi-structured interviews with patients $(n=60)$ 
seeking health services in either a sub-acute area (an area designed to respond to patients triaged as stable and non-urgent) of a large urban Emergency Department (ED), or a community health center (CHC); (d) interviews with 16 healthcare workers who were involved in either administering an ethnic identity question in healthcare agencies, or whose agencies were considering doing so as part of intake data. Data were collected between February 2006 and August 2007.

Ethical approval was obtained from the University Research Ethics Board. Patients were recruited in the waiting room of the ED or CHC. They were approached initially by a unit clerk. If interested, they were directed to a trained research assistant who explained the study fully and, if consent was obtained, conducted the interview. All other participants were recruited by word of mouth through our research partners in the Health Authority. Interviews focused on their thoughts regarding people being asked to identify their ethnicity in healthcare settings, past experiences with being asked, the benefits that might be gained from such information and their possible concerns. Interviews were audio-recorded and transcribed. Field notes recorded appearance, non-verbal behaviour and tones of voice during interviews.

The policy decision-makers/leaders included those responsible for patient information systems, Aboriginal health and "diversity" portfolios in the Health Authorities and those associated with policy research units dealing with health disparities. The focus group participants included: (a) Focus Group \#1 - community leaders who self-identified as "visible minorities", primarily as East Indian or Chinese people. In Canada, visible minorities are defined in racializing terms as "persons who are...non-Caucasian in race or non-white in Colour...Aboriginal persons are not considered to be members of visible minority groups" (Statistics Canada, 2006); (b) Focus Group \#2A and B - two groups of community leaders who self-identified as Aboriginal, the term used to refer generally to the indigenous inhabitants of Canada including First Nations, Métis and Inuit peoples (Royal Commission on Aboriginal Peoples, 1996, p. xii). This included both status and non-status people, with "status" denoting those people registered with the federal government's Indian Registry.

The patients we interviewed included 22 people who selfidentified as members of an Aboriginal group, and 17 who identified as English-speaking Euro-Canadians. The remaining 21 patients self-identified as members of various other ethno-cultural groups. These included people who would be defined as visible minorities having immigrated or descended from immigrants from places such as India, Taiwan, South America, Turkey, and people who would not be so defined who were recent immigrants from places such as Eastern Europe.

An interpretive thematic analysis was conducted. Each transcript and associated field notes were read to get a sense of the whole and then coded thematically. Themes were compared across interviews and revised. We used a qualitative software package (NVivo ${ }^{\mathrm{TM}}$ ) to manage data and organize preliminary coding. This paper presents our analysis of the data using an ethical lens as our theoretical perspective.

\section{An ethical lens}

Ethics are concerned with values and morality. However, as feminist and contextual ethicists (e.g. Sherwin, 1992; Walker, 2001) argue, ethics "is fundamentally, a discourse about morality and power" (Walker, 2001, p. 4). An ethical lens that considers power was useful because of our interest in critical inquiry and need to scrutinize ideological assumptions within our own analysis and the practices under study. Inherent in the quest to understand the implications of collecting ethnicity data was the desire to optimize the benefits and avoid harm. Such questions are ethical questions. Further, the research addresses contexts of power and the social positions of patients within existing power structures - patients are to be asked certain questions within social structures and organizational systems in which power differentials operate with particular effects. Patients coming for healthcare are often vulnerable due to illness, disability and social positioning within the healthcare system. These vulnerabilities are compounded by racializing and marginalizing practices in healthcare. Finally, the implications of collecting ethnicity data are of considerable moral concern because they involve categorization, racializing and in some cases, discriminatory processes. While discrimination may simply mean 'to distinguish between', as Lippert-Rasmussen argues "discrimination is bad, when it is, because it harms people" [emphasis added] (2006, p. 167). Thus, an ethical analysis of the approaches to asking about ethnicity must address the extent to which the practices involved (which may include not collecting such data) may harm people.

The concept of harm is central to ethics. However, what is meant by harm varies. Within biomedical ethics the caveat "First, do no harm" - for example as articulated within the mid-range principles proposed Beauchamp and Childress (2001) - is interpreted primarily to mean that one should not cause harm to others in the course of delivering healthcare. The emphasis is on physical harm caused by individual providers in the process of giving care to individual clients (Pauly, 2008). However, within bioethics, rarely are wider contexts and preventing harm considered or seen as a priority. Through the principle of autonomy, individual liberty is stressed and freedom of choice implicitly understood as the mechanism by which individuals protect their interests and mitigate harm, a perspective that overlooks power dynamics. For example, some proponents of ethnicity data collection argue that harm is mitigated because individual patients have the choice whether or not to answer an ethnicity question. Such understanding does not account for the pressures and vulnerabilities patients may experience when seeking care and how such pressures may affect their capacity to exercise choice. Thus, in concert with trends within procedural justice research, as Sandefur (2008) argues, an important approach to inequality and access to justice come from research that explores people's subjective evaluations of their experiences.

From a broader theoretical perspective, harm can be understood as the consequence of a wide range of circumstances or actions beyond those of particular individuals. Feminist philosophers such as Young (1990) draw attention to the harms associated with belonging to certain groups and experiencing certain conditions. To consider the implications of collecting ethnicity data, analysis of possible harms associated with being assigned to or being seen as belonging to particular ethnic groups is required. Thus, we understood harm to refer to damage that might ensue from a wide range of social structures and practices, while focusing on practices related to collecting ethnicity data.

\section{Findings}

The findings of this study surface tensions between good intended by collecting and using ethnicity data, and the harms that may be incurred through the process of collecting and using data.

\section{Possible benefits and good intentions}

I'm assuming they would use that information for good purposes (Patient \#44) 
A range of possible benefits of ethnicity data were imagined. Policy decision-makers/leaders and healthcare workers generally were more positive than community leaders and patients, and focused upon the potential uses of having ethnicity data (rather than on the processes used to obtain that data) to better understand patterns among groups. The advantages fell into three overlapping areas that participants appeared to see as being causally linked: a) the advantage of being able to map the ethnicity of the patient populations (determining who is accessing care), b) the possibility of identifying barriers to care based on ethnicity, and c) the opportunity to make care better and more equitable, primarily by better tailoring services to meet the needs of diverse ethnocultural groups. Decision-makers articulated these benefits most clearly:

"The main reason for collecting ethnicity data or any kind of ethnic coding is to identify and therefore be able to develop strategies to address health disparities" Decision-maker \#10

"If we can't measure disparities in either their health status, access to services, types of treatment prescribed, health outcomes, it is an invisible problem" Decision-maker \#6

Albeit to a lesser extent, some community leaders and several patients identified similar potential benefits for groups. Some patients supposed that ethnicity data might be used to ameliorate health disparities and racism and made assumptions similar to those made by policy decision-makers/leaders that data collection would lead to action. For example, Patient \#1, who was Canadianborn, referred to himself as "black", and identified as "Canadian", thought the collection of such data could hypothetically convey to people in healthcare "that racism is being watched and monitored, and they will be held accountable for any wrongful action..." Some patients expressed a sense of faith in healthcare as an essentially equitable system, and therefore saw the collection of ethnicity data as innocuous. As Patient \#44, who identified as EuroCanadian, said "Well, because we live in Canada and we're such a mixed culture...everybody is kind of equal so I don't think anyone specifically is being targeted for anything [in healthcare]". Further, patients often expressed implicit trust that data would be used for a good purpose, and that if it was being requested, it must be necessary. Patient \#50, who identified as Pilipino said "I would readily give away where I'm from if that would help the medical team resolve the problem". Patient \#31 who identified as Aboriginal (Nuxalk and Métis) offered: "I mean, if they have a panel of all different ethnics [referring to leaders in healthcare], sure, let them [use the data]".

Patients did not emphasize benefits and only occasionally suggested benefits for groups, instead focusing on the effects on particular individuals being asked. They generally associated possible advantages with knowing a person's country of origin, primary language, or presumed genetic makeup, and connected that knowledge with some anticipated benefit in terms of better clinical care for that person. For example, Patient \#2 who identified as a German-American Canadian said "if you come from a poorer country...you hear people from Africa, their water supply is pretty bad over there, so...it could have an effect on health, so...I think the doctor needs to know...for better treatment, right?"

The primary benefit identified by both patients and decisionmakers was that having ethnicity data might ensure that clinical care would take into account risk factors associated with health problems that they, sometimes erroneously, thought were biologically or genetically linked. Reflecting popularized discourses in Canadian society (and other countries) that conflate ethnicity with biological/genetic notions of race, ethnicity was understood as a means through which to identify biologically based risk factors. ...for instance Asians are more prone to liver cancer, Japanese have very high instances of stomach cancer... Focus Group \#1

"There are certain groups in the population that might be prone to certain diseases based on your ethnicity, so I guess it goes back to genetics" Patient \#1 (Canadian)

"The only need for that information would be genetic, and you know, derivatives of perhaps your genotype that would be susceptible..." Patient \#18 (Métis)

"Your roots, like they could look back into your family history and get you the proper healthcare" Patient \#20 (Euro-Canadian)

Participants' assumptions mirrored popularized, though erroneous, assumptions about the genetic basis of disease patterns, and often conflated the notion of family history as an easily identifiable risk factor, with presumed genetic relatedness among ethnocultural groups. Participants thought that knowing ethnicity might explain why people have trouble taking their prescribed medications, or could help with identifying what people eat. Several thought there would be advantages to collecting ethnicity data if the person did not speak English. However, as we continue to discuss, ethnicity as a single variable collected at the point of care is unlikely to yield this type of information, which could be better obtained by directly asking about family history, languages spoken or diet preferences.

Perceptions of benefits (and harms) were related to participants' own identities, social and historical locations, and experiences. For example, Patient \#31 related a positive healthcare experience that she associated with the physician knowing about her ethnicity.

I had a couple of doctors that did know native background and he asked me where I was from and automatically he knew what kind of [pause] he asked me these questions, like there's heart disease and arthritis, and there's a kind of blood type we get in this area... and I have one of them. And he seemed to understand more... and right away I got the help I needed instead of doing all the run around.

Those who self-identified as 'white', Euro-Canadian or Caucasian, tended to see no benefit to reporting ethnicity for themselves. Patient \#36 thought there was no benefit "because I am part of the mainstream". Patient \#44 said "For me specifically it probably won't benefit me that much because I was born and raised in Canada, and so I am quite fluent in English..." If they imagined benefits, then they associated the benefits with people who have immigrated to Canada, are not fluent in English or could be classified as visible minorities. Similarly, they did not tend to see potential harms as applying to themselves. For example, Patient \#35 said, "I am a Canadian white guy, so it doesn't bother me at all".

\section{Harms and concerns}

"What do you mean, what am I? I'm here to get treatment, is what I am, right?" Patient \#21

At the same time as suggestions were offered regarding the potential benefits, primarily of having ethnicity data and serving groups better, the overwhelming response to the idea of the process of asking about ethnicity was deep concern. Patients and community leaders raised these concerns emphatically. Because we conducted patient interviews in an Emergency unit and a community healthcare center, patients were ideally positioned to consider data collection in a clinical context. In contrast to asking about the experience more theoretically in surveys, patients in waiting rooms could imagine the experience more directly. As when considering benefits, participants identified harms by drawing on their own 
identities, experiences, social positions, and experiences of discrimination.

Across focus group and patient participants, the harms anticipated included being judged on the basis of assumptions and stereotypes, and the possibility of receiving poorer care based on such judgements. While some benefits were associated with groups, participants anticipated harmful effects primarily for individuals. Participants expressed their concerns in relation to groups they thought likely to be vulnerable to the effects of inequities and racialization, such as Aboriginal people and visible minorities. Many were concerned that ethnicity data could influence healthcare staff to reinforce stereotypes that link certain health behaviours to particular groups - for example, the commonly-held stereotype in Canada that links Aboriginal people to alcoholism (Furniss, 1999). Many who self-identified as 'white', Euro-Canadian or Caucasian expressed concern, not for themselves, but for those they saw as targets of negative judgement. Patient \#36 said

"just imagine if you had a physician or a nurse that didn't like Aboriginal people or didn't like Indo Canadians or didn't like new immigrants...I worked a lot with Aboriginal people, so the issue for them is, can the data be used against them?"

Implicit was that healthcare and social inequities are prevalent. Adding questions related to one's ethno-cultural background was viewed as a process that would fuel anxieties about ongoing inequities, and how inequities could manifest in healthcare contexts because of negative perceptions or assumptions that some staff may have toward particular groups. Several patients said that they would not answer ethnicity questions. Patient \#26, who identified as "Canadian" and appeared Caucasian, said "IIf] I thought they were going to treat me differently, I'd probably lie to them". Patient \#7 who identified as "Ismaili" said "It depends who is working behind the desk and what kind of attitude [they have]...I think a lot of people are just racist...They've got the wrong people working behind the desk often".

Of great concern was the extent to which those who identified as members of a visible minority or as Aboriginal expressed concern for themselves. When asked their thoughts regarding ethnicity being collected as part of routine patient information at the point of care, many expressed anxiety, fear and anger. Patients who would be identified as "visible minorities" said:

I feel extremely highly discriminated towards by asking such a question. Patient \#48

I'd be [offended] if this was asked of me. It's just another means to divide. Patient \#21

Because its, its, its not a good question, its not a good question at all, it doesn't relate to my health, this sort of question, it makes me really angry. Patient \#41

Concerns extended beyond the stress of being asked to fear that such questions both signalled and could lead to further discrimination and poorer care. Patient \#21, explained

...being a black man... I have a tendency to be kind of squeamish about any type of questions... and my first thought when you ask me that coming into an emergency... if I was kind of bleeding and everything else and they asked me well where am I from, okay, I might assume that they're thinking well this is an African, you know, they're paranoid of getting AIDS or something else.

Participants who identified as Aboriginal expressed similar concerns.

I would feel insulted, offended, marginalized, targeted. I would wonder how this is going to affect my treatment, I would wonder how this would affect nurses', doctors' behaviour towards me. My first response would probably be "what difference does it make?" So I would be on the offensive. I feel that almost, even as you asked the question, like how would I feel? I feel something rise up in me, to just really... and it makes me angry, immediately. And that's just in this room. I can't even imagine how I would feel if I was hurt or if I was bringing in my husband who is dead or dying or whatever, and then to be asked if I was, what are you? I think I'd probably go through [pause] I wouldn't be able to behave properly, probably. Focus Group \#2B

Each participant who identified as Aboriginal described the effects of discrimination on his or her health. For example, Patient \#31 described how the stereotypical idea that Aboriginal people sell their pain medications not only can create problems for pain management, but also may create or exacerbate the problem of street sale of prescription drugs:

A lot of us have arthritis, and... are being put down from Tylenol 3's to Tylenol 2's, then to regular Tylenol because [doctors] don't want to hand it out, they think we are selling them. In other words we have to go out and buy our medications off the street sometimes.

The idea that "Aboriginality" could be interpreted as a risk factor for health or social problems was identified by several participants and echoed Smylie's (2005) concerns about the risks of pathologizing "Aboriginality" itself as a risk factor in healthcare contexts.

Importantly, patients and focus group members were concerned that inequities played out along the intersecting axes of class, culture, race and ethnicity. Patient \#1 who referred to himself as "black", and identified as Canadian, explained

...If they respect you more because you're rich...they'll treat you with more respect...that's why, about ethnicity, um, about your race rather, ...I would feel right away, I mean being classified as being prejudged and people would treat me accordingly.

Most participants who identified as Aboriginal discussed these intersections to some extent. For example, Patient \#31 spoke at length regarding her different experiences of accessing care depending on how she was dressed. She explained how her family carefully planned their appearances when accessing healthcare, arguing that class assumptions were at least as powerful as those based on ethnicity. Similarly, Patient \#56 described how his treatment differed when he was able to shower in contrast to when he was living on the street and unable to do so. These concerns reflect anxieties that Aboriginal people have expressed in other studies about the need to transform one's appearance when seeking healthcare as a means of gaining legitimacy and credibility as a medical subject (e.g. Tang \& Browne, 2008).

Patients, focus group participants and some healthcare leaders identified concerns based on harmful discrimination that they had either experienced directly or witnessed - both within healthcare and wider social interactions. For example, in Focus Group \#2A, one woman associated her daughter being treated rudely and her own experience of having her pain ignored with being identified as Aboriginal. Participants drew upon experiences of racial and class discrimination, most often as race, ethnicity and class intersected. Patient \#13, who identified as Métis, recounted observing how a man was treated in Emergency.

He was bleeding all over the place and they just ignored him. You could see he needed more treatment...he was the one that needed the help the most and he was the last one they seen...he looked like a bum off the street so...they put the high class first, like, the ones that look you know, well off.

His experiences fuelled his concern that indigent patients likely would be treated differentially regardless of need - which fed his 
worry about how he might be treated on the basis of his perceived socioeconomic status and ethnicity. Similarly, an admitting clerk responsible for collecting ethnicity data had considerable caution about doing so because of numerous personal experiences such as the following;

my brother had a head injury...slipped on the ice and he and my brother had been hunting and they went back to a cabin and were sharing a bottle of wine with people and there was alcohol on his breath and they medivaced [him] from the hunting area and they treated him like another drunk Indian because of the fact that he was acting violently and he's not a violent person, my brother was begging them, "this is not my brother['s normal behaviour] and there's something wrong..." and it was the head injury...it took twenty-four hours for them to [transport] him to Vancouver. Healthcare Worker \#3

Patients and focus group participants frequently linked their concerns about being asked their ethnicity to questions about how the information might be used - and for what aims. Thus, in addition to anticipating harm to individuals, and in contrast to the explicit trust expressed by some, many participants of all identities questioned the usefulness of ethnicity data, including how and whether such data might be used beneficially. Patient \#24 who identified as 'white' said '[I'm] concerned, well, yeah, curious as to why...what's the data being used for...?" Similarly, participants in Focus Group \#1 expressed caution.

I guess it really depends on the context within which you do ask. Is it for the purpose of diagnosing? So if you're Chinese you might have some idea of your diagnosis. Whereas if you were asked at a walk-in [clinic] or emergency and you are asked what ethnicity you are, it's like, well, "what difference does it make? I'm here because I need help!"

\section{Patient \#32 who identified as "First Nations" said}

I'm just afraid that they might put a, our ethnicity [as] more vulnerable to diseases which I don't think is true, right, and I'm afraid they might put more onto that and...it will be used against us sometime in the future...

Similarly, Patient \#6, who identified her ethnicity as Roman Catholic (and whose first language was Portuguese), said

Particularly in the future if the Health Authority or the federal government started asking people for their ethnic origin, because it separates the people based on color of skin or where they're from, then we can't call ourselves a society. Just can't.

Participants expressed overall caution about collecting ethnicity data. For example, Patient \#11 identified as Iranian-Canadian, and although he had "no difficulty" sharing his ethnicity and expressed trust in healthcare providers, he said:

I hope in a naive manner that everybody who comes in or works in a hospital, all the physicians, would have the best interest of the patient in mind... and not all the time that would be true so it's a really sensitive issue and should be really scrutinized before being implemented.

\section{Discussion}

In contrast to perceptions of Canada as egalitarian and equitable, and despite universal healthcare, health and healthcare inequities are significant and persistent, and experiences of racialization and discrimination routinely shape peoples' access to and utilization of services (e.g. Anderson \& Kirkham, 1998). This exploratory study sheds light on the complexity of issues at play in Canadian healthcare settings in relation to asking people to identify their ethnicity at the point of care. More research is needed to more fully understand the complex range of issues involved, particularly as patients receiving care in other healthcare locations may have different views than the participants in this study. However, these findings provide evidence for why there can be distress generated in the process of self-categorization of ethnicity and widespread inaccuracy of data within clinical contexts. Through the analysis it became evident that the collection of ethnicity data represented a racializing process for many patients, with potentially harmful effects.

Discrimination is used in relation to ethnicity, culture, and race in two senses: "racialism, which is the not necessarily objectionable view that the human race is divided into different, biologically real races, and racism, which is the objectionable view that this supposedly biologically real division involves a hierarchy of value" (Lippert-Rasmussen, 2006, p. 168). The collection of ethnicity data presumably involves a well-intentioned implementation of racialism that is inevitably perpetuated because of the conflation of race and ethnicity. However, the findings from this study suggest that it is not possible to implement collection of ethnicity data at the point of care (on the basis of racialism) without invoking anxieties about racism and racist classifications - particularly among people who have experienced racialization and healthcare inequities. Despite the ethically-motivated intentions of Health Authorities in Canada to redress health and healthcare inequities by collecting ethnicity data at the point of care, current levels of inequities, and their disproportionate effects on particular groups of people, preclude any "neat" separation of racialism and racialization.

The findings highlight that concerns about harm must be taken seriously. These harms are not simply perceptions and the harms of racializing processes are not limited to the psychological effects of discrimination. Rather, as Stuber, Meyer, and Link (2008) recently argued, discrimination is thought to be health harming through various pathways including a) the direct effects of stress arising from interactions that are perceived to be discriminatory, b) denial of access to resources, c) internalization of stigma and discrimination, and d) vigilance in anticipation of negative treatment leading to stress and impaired social interactions between marginalized and non-marginalized persons. Discriminatory assumptions can have significant effects on a range of decisions and the allocation of healthcare resources (King et al., 2008; van Ryn \& Fu, 2003). For example, in the United Kingdom, Balsa et al. (2005) found that racialized assumptions made by physicians even before observing any particular signal from the patient, and communication problems between "white" physicians and minority patients affected diagnostic decisions to the point that these effects could account for "racial" or ethnic group differences in the diagnosis of hypertension and diabetes. Further, the collection of such data may contribute to reluctance by some to access care. For example, in the US, Baker et al. (2005) found that $18.5 \%$ of African Americans and $26.3 \%$ of Hispanics said they would be less likely to go to a hospital or clinic that collected information about race and ethnicity, suggesting such connections should be further explored.

While aggressive action to address health inequities is urgent, given available population and research evidence of inequities and lack of evidence of action based on clinically collected ethnicity data, it is questionable whether individual level ethnicity data collected in a Health Authority - as an isolated variable of interest are necessary to increasing equitable care. Our results suggest that collecting ethnicity data in a clinical context is not a neutral process. The potential harms to individuals are considerable and include harms that may directly contribute to exacerbating 
inequities and serve as barriers to healthcare access. Such practices must be evaluated in the context of increasing politics of fear, racial profiling and growing inequities and alternatives sought. These concerns lead us to recommend that, in the current climate of healthcare, evidence is required regarding the benefits of collecting ethnicity at the point of care before the widespread adoption of such action. This study clearly supports Pringle and Rothera's (1996) similar conclusion in the United Kingdom regarding general practice - clearer evidence of benefit is required before asking about ethnicity can be recommended. If, despite these concerns, healthcare organizations in Canada pursue collection of ethnicity data in clinical contexts, then care should be taken to mitigate harms at individual and group levels. Collection methods should be considered carefully. Given evidence of preferences for computerbased, or paper and pencil responses to screening for violence (e.g. MacMillan et al., 2006), these alternatives should be evaluated. For face-to-face data collection, strategies to mitigate harm, including extensive training of data collectors, will be required. Even when protocols regarding ethnicity data collection call for self-identification, admitting clerks often "assign" ethnicity based on appearances (Gomez, Kelsey, Glaser, Lee, \& Sidney, 2005; Hasnain-Wynia \& Baker, 2006). Reasons for collecting ethnicity data should be clearly articulated by healthcare organizations and communicated to both care providers and patients, and commitment to action related to those reasons made and enacted.

Most importantly, perceived discrimination and the issues underlying such discrimination in healthcare settings must be taken seriously and addressed at the system level. The assumption should be challenged that providing equitable, quality care in the clinical context requires the collection of race and ethnicity data (particularly at the point of care), and strategies for providing equitable care should be pursued actively. Indeed, actions to address structural inequities should be taken immediately at healthcare levels and beyond (Smedley, 2008; Smedley, Rich, \& Erb, 2005).

\section{Acknowledgements}

The research reported in this study was funded by the Michael Smith Foundation for Health Research.

The authors would like to acknowledge the important contributions of the people we interviewed, and Dr. Koushambhi Basu Kahn, Ms. Laurel Jebamani and Ms. Tej Sandhu for their assistance in data collection and analysis. We would also like to acknowledge the leadership provided by the co-investigators on our research team: Dr. Betty Calam, Department of Family Practice, Faculty of Medicine; Ms. Nadine Caplette, Vancouver Coastal Health (until April 2008); Mr. Ron Peters, Vancouver Coastal Health; Ms. Elizabeth Stanger, Vancouver Coastal Health.

\section{References}

Anderson, J., \& Kirkham, S. R. (1998). Constructing nation: the gendering and racializing of the Canadian healthcare system. In V. Strong-Boag, S. Grace, A. Eisenberg, \& J. Anderson (Eds.), Painting the maple: Essays on race, gender, and the construction of Canada (pp. 242-261). Vancouver, BC: University of British Columbia Press.

Aspinall, P., \& Anionwu, E. (2002). The role of ethnic monitoring in mainstreaming race equality and the modernization of the NHS: a neglected agenda? Critical Public Health, 12(1), 1-15.

Aspinall, P. J. (2000). The mandatory collection of data on ethnic group of inpatients: experience of NHS trusts in England in the first reporting years. Public Health, 114(4), 254-259.

Baker, D. W., Cameron, K. A., Feinglass, J., Georgas, P., Foster, S., Pierce, D., et al. (2005). Patients' attitudes toward healthcare providers collecting information about their race and ethnicity. Journal of General Internal Medicine, 20(10), 895-900.

Balsa, A. I., McGuire, T. G., \& Meredith, L. S. (2005). Testing for statistical discrimination in health care. Health Services Research, 40(1), 227-251.
Beauchamp, T. L., \& Childress, J. F. (2001). Principles of biomedical ethics. New York: Oxford University Press.

Bhopal, R. (1998). Spectre of racism in health and healthcare: lessons from history and the United States. British Medical Journal, 316(7149), 1970-1974.

Bhopal, R. (2001). Ethnicity and race as epidemiological variables: centrality of purpose and context. In H. Macbeth, \& P. Shetty (Eds.), Health and ethnicity (pp. 21-40). London: Taylor \& Francis.

Bhopal, R. (2007). Racism in health and healthcare in Europe: reality or mirage? European Journal of Public Health, 17(3), 238-241.

Bourassa, C., McKay-McNabb, K., \& Hampton, M. R. (2004). Racism, sexism, and colonialism: the impact on the health of Aboriginal women in Canada. Canadian Woman Studies, 24(1), 23-29.

Bourhis, R. Y. (2003). Measuring ethnocultural diversity using the Canadian census. Canadian Ethnic Studies, 35(1), 9-33.

Ford, M. E., \& Kelly, P. A. (2005). Conceptualizing and categorizing race and ethnicity in health services research. Health Services Research, 40(5), 1658-1675.

Furniss, E. (1999). The burden of history: Colonialism and the frontier myth in a rural Canadian community. Vancouver, BC, Canada: University of British Columbia Press.

Gerrish, K. (2000). Researching ethnic diversity in the British NHS: methodological and practical concerns. Journal of Advanced Nursing, 31(4), 918-925.

Gomez, S., Kelsey, J., Glaser, S., Lee, M., \& Sidney, S. (2005). Inconsistencies between self-reported ethnicity and ethnicity recorded in a health maintenance organization. Annals of Epidemiology, 15(1), 71-79.

Hasnain-Wynia, R., \& Baker, D. W. (2006). Obtaining data on patient race, ethnicity, and primary language in healthcare organizations: current challenges and proposed solutions. Health Services Research, 41, 1501-1518.

Hasnain-Wynia, R., Pierce, D., \& Pittman, M. (2004). Who, when, and how: The current state of race, ethnicity, and primary language data collection in hospitals. New York: The Commonwealth Fund.

Henry, F., \& Tator, C. (2006). The colour of democracy: Racism in Canadian society. Toronto, ON: Nelson.

Jedwab, J. (2003). Coming to our census: the need for continued inquiry into Canadian's ethnic. Canadian Ethnic Studies, 35(1), 33-51.

Kaplan, J. B., \& Bennett, T. (2003). Use of race and ethnicity in biomedical publication. JAMA: Journal of the American Medical Association, 289(20), 2709-2716.

King, R. K., Green, A. R., Tan-McGrory, A., Donahue, E. J., Kimbrough-Sugick, J., \& Betancourt, J. R. (2008). A plan for action: key perspectives from the racial/ ethnic disparities strategy forum. Milbank Quarterly, 86(2), 241-272.

Krieger, N. (1999a). Embodying inequality: a review of concepts, measures, and methods for studying health consequences of discrimination. International Journal of Health Services, 29(2), 295-352.

Krieger, N. (1999b). "Whiting out" white privilege will not advance the study of how racism harms health. American Journal of Public Health, 89(5), 782-783.

Krieger, N. (2000). Refiguring "race": epidemiology, racialized biology, and biological expressions of race relations. International Journal of Health Services, 30(1), 211-216.

Krieger, N. (2004). Data, “race," and politics: a commentary on the epidemiological significance of California's proposition 54. Journal of Epidemiology and Community Health, 58(8), 632-633.

Krieger, N., Chen, J. T., Waterman, P. D., Rehkopf, D. H., \& Subramanian, S. V. (2005). Painting a truer picture of US socioeconomic and racial/ethnic health inequalities: The Public Health Disparities Geocoding Project. American Journal of Public health, 95(2), 312-323.

Lippert-Rasmussen, K. (2006). The badness of discrimination. Ethical Theory $\mathcal{E}$ Moral Practice, 9(2), 167-185.

MacMillan, H. L., Wathen, C. N., Jamieson, E., Boyle, M., McNutt, L.-A., Worster, A., et al. (2006). Approaches to screening for intimate partner violence in healthcare settings. JAMA: Journal of the American Medical Association, 296(5), 530-536.

National Center for Health Statistics. (2008). National health interview survey 1957-2007.

New Zealand Ministry of Health. (2004). Ethnicity data protocols for the health and disability sector. (p. 32). Wellington, New Zealand: New Zealand Ministry of Health.

Pauly, B. (2008). Harm reduction through a social justice lens. International Journal of Drug Policy, 19(1), 4-10.

Perot, T., \& Youdelman, M. (2001). Racial, ethnic, and primary language data collection in the healthcare system: An assessment of federal policies and practices. ( $\mathrm{p}$. 40). New York: The Commonwealth Fund.

Physicians for Human Rights. (2003). The right to equal treatment: A report by the panel on racial and ethnic disparities in medical care by Physicians for Human Rights. Washington, DC: Physicians for Human Rights.

Pringle, M., \& Rothera, I. (1996). Practicality of recording patient ethnicity in general practice: descriptive intervention study and attitude survey. BMJ: British Medical Journal, 312(7038), 1080-1082.

Quan, H., Wong, A., Johnson, D., \& Ghali, W. (2006). The public endorses collection of ethnicity information in hospital: implications for routine data capture in Canadian health systems. Healthcare Policy, 1(3), 55-64.

Royal Commission on Aboriginal Peoples. (1996). Report of the Royal Commission on Aboriginal Peoples. Ottawa: Canada Communications Group-Publishing.

Rummens, J. A. (2003). Ethnic ancestry, culture, identity, and health: using ethnic origin data from the 2001 Canadian census. Canadian Ethnic Studies, 35(1), 84-110.

van Ryn, M., \& Fu, S. S. (2003). Racial/ethnic bias and health. Paved with good intentions: do public health and human service providers contribute to racial/ ethnic disparities in health? American Journal of Public Health, 93(2), 248-255.

Sandefur, R. L. (2008). Access to civil justice and race, class, and gender inequality. Annual Review of Sociology, 34(1), 339-358. 
Sherwin, S. (1992). Feminist and medical ethics: two different approaches to contextual ethics. In H. Bequaret Holmes, \& L. Purdy (Eds.), Feminist perspectives in medical ethics (pp. 17-31). Indianapolis: Indiana University.

Sheth, T., Nargundkar, M., Chagani, K., Anand, S., Nair, C., \& Yusuf, S. (1997). Classifying ethnicity utilizing the Canadian mortality database. Ethnicity E Health, 2(4), 287-295.

Smedley, B. D. (2008). Moving beyond access: achieving equity in state healthcare reform. Health Affairs (Project Hope), 27(2), 447-455.

Smedley, B. D., Rich, R. F., \& Erb, C. T. (2005). Racial and ethnic healthcare disparities: the role of personal choice vs. structural inequality. In R. F. Rich, \& C. T. Erb (Eds.), Consumer choice: Social welfare and health policy - Policy studies review annual (pp. 83-98). New Brunswick, N.J. and London: Transaction.

Institute of Medicine Committee on Understanding and Eliminating Racial and Ethnic Disparities in Healthcare. In Smedley, B. D., Stith, A. Y., \& Nelson, A. R. (Eds.), Unequal treatment: Confronting racial and ethnic disparities in healthcare. Washington, D.C.: Institute of Medicine National Academies Press.

Smylie, J. (2005). The ethics of research involving Canada's Aboriginal populations. Canadian Medical Association Journal, 172(8), 977.
Statistics Canada. (2006). Definitions, data sources and methods. Ottawa: Statistics Canada.

Stuber, J., Meyer, I., \& Link, B. (2008). Stigma, prejudice, discrimination and health. Social Science \& Medicine, 67(3), 351-357.

Tang, S. Y., \& Browne, A. J. (2008). 'Race' matters: racialization and egalitarian discourses involving aboriginal people. Ethnicity \& Health, 13(2), 1-19.

Thomas, D. (2005). I am Canadian. Canadian Social Trend 1-7.

United Nations. (1952). The race question in modern science: The results of an inquiry - The concept of race. Paris: United Nations.

Walker, M. U. (2001). Seeing power in morality: a proposal for feminist naturalism in ethics. In P. DesAutels, \& J. Waugh (Eds.), Feminists doing ethics. Oxford, London: Rowman \& Littlefield.

Williams, D. R. (2002). Racial/ethnic variations in women's health: the social embeddedness of health. American Journal of Public Health, 92(4), 588-597.

Wu, Z., \& Schimmele, C. M. (2005). Racial/ethnic variation in functional and selfreported health. American Journal of Public Health, 95(4), 710-716.

Young, I. M. (1990). Justice and the politics of difference. Princeton, NJ: Princeton University Press. 